\title{
Análise da ocorrência de reações adversas à poliquimioterapia no tratamento para hanseníase
}

\author{
Analysis of the occurrence of adverse reactions to polychemotherapy in the treatment of \\ leprosy
}
Análisis de la aparición de reacciones adversas a la poliquimioterapia en el tratamiento de la lepra

Carla Andréa Avelar Pires ${ }^{1 *}$, Maria Amélia Lopes dos Santos ${ }^{1}$, Beatriz Hosana Biasi ${ }^{1}$, Amanda Gabay Moreira1, Amália Costa Coimbra ${ }^{1}$, Mariana Chisté Ferreira ${ }^{1}$, Mayara Silva Nascimento ${ }^{1}$, Juliana Bacellar Nunes de Brito ${ }^{1}$, Francisca Regina Oliveira Carneiro' ${ }^{1}$.

\section{RESUMO}

Objetivo: Analisar os principais efeitos colaterais do esquema medicamentoso padrão, poliquimioterapia, utilizada no tratamento para hanseníase. Métodos: Trata-se de estudo transversal descritivo, conduzido com 40 participantes, atendidos no Ambulatório de Dermatologia da Universidade do Estado do Pará. Os pesquisadores empregaram uma adaptação da Ficha para Investigação de Ocorrência de Efeitos Adversos na Poliquimioterapia (PQT) em hanseníase, com variáveis agrupadas em perfil social, dermatológico, laboratorial e reacional. Resultados: Os pacientes em sua maioria foram: homens, com mais de 60 anos de idade, aposentados, com apenas ensino fundamental. Quanto a classificação clínica, predominantemente, a forma dimorfa e multibacilar, em 12 meses de tratamento. Os sistemas orgânicos com maior relação com reações adversas foram: excretor, nervoso, tegumentar e respiratório, sobretudo mediante ao uso da dapsona. Entre os efeitos adversos, destaca-se o enjoo (32,50\%) no uso de rifampicina, mialgia (65\%) no uso de dapsona e hiperpigmentação cutânea $(77,50 \%)$ no uso de clofazimina. Conclusão: $O$ presente estudo demonstrou que as reações adversas mais frequentes foram leves e estão associadas ao uso de dapsona e clofazimina, sendo de suma importância a detecção precoce desses efeitos, para eficaz intervenção de eventos graves.

Palavras-chave: Hanseníase, Reações adversas relacionados a medicamentos, Poliquimioterapia.

\section{ABSTRACT}

Objective: To analyze the main side effects of the standard drug regimen, polychemotherapy, used in the treatment of leprosy. Methods: This is a descriptive cross-sectional study, conducted with 40 participants, attended at the Dermatology Clinic of the University of the State of Pará. The researchers used an adaptation of the Form for Investigation of the Occurrence of Adverse Effects in Polychemotherapy (PQT) in leprosy, with variables grouped into social, dermatological, laboratory and reaction profiles. Results: Most patients were: men, over 60 years of age, retired, with only elementary education. As for the clinical classification, predominantly, the dimorphic and multibacillary form, in 12 months of treatment. The organic systems most closely related to adverse reactions were: excretory, nervous, cutaneous and respiratory, especially through the use of dapsone. Among the adverse effects, nausea $(32.50 \%)$ in the use of rifampicin, myalgia $(65 \%)$ in the use of dapsone and skin hyperpigmentation (77.50\%) in the use of clofazimine stands out. Conclusion: The present study demonstrated that the most frequent adverse reactions were mild and are associated with the use of dapsone and clofazimine, and the early detection of these effects is extremely important for the effective intervention of serious events.

Keywords: Leprosy, Drug-related side effects, Drug therapy.

\section{RESUMEN}

Objetivo: Analizar los principales efectos secundarios del régimen farmacológico estándar, la poliquimioterapia, utilizado en el tratamiento de la lepra. Métodos: Se trata de un estudio descriptivo, transversal, realizado con 40 participantes, atendido en la Clínica de Dermatología de la Universidad del

1 Universidade do Estado do Pará (UEPA), Belém - PA. *E-mail: carlaavelarpires@gmail.com 
Estado de Pará. Los investigadores utilizaron una adaptación del Formulario de Investigación de la Ocurrencia de Efectos Adversos en Poliquimioterapia (PQT) en lepra, con variables agrupadas en perfiles sociales, dermatológicos, de laboratorio y de reacción. Resultados: La mayoría de los pacientes fueron: hombres, mayores de 60 años, jubilados, con solo educación básica. En cuanto a la clasificación clínica, predomina la forma dimórfica y multibacilar, en 12 meses de tratamiento. Los sistemas orgánicos más relacionados con las reacciones adversas fueron: excretor, nervioso, cutáneo y respiratorio, especialmente mediante el uso de dapsona. Entre los efectos adversos destacan náuseas (32,50\%) en el uso de rifampicina, mialgia (65\%) en el uso de dapsona e hiperpigmentación cutánea $(77,50 \%)$ en el uso de clofazimina. Conclusión: El presente estudio demostró que las reacciones adversas más frecuentes fueron leves y están asociadas al uso de dapsona y clofazimina, siendo la detección temprana de estos efectos de suma importancia para la intervención efectiva de eventos graves.

Palabras clave: Lepra, Reacciones adversas relacionados con medicamentos, Quimioterapia combinada.

\section{INTRODUÇÃO}

A hanseníase ainda é uma doença bastante prevalente mundialmente, com mais de 200 mil casos novos da doença em 2018, considerada endêmica nas áreas em desenvolvimento da Ásia, da África e da América Latina (BRASIL, 2020; PODDER I, et al., 2018). É uma doença infectocontagiosa crônica de evolução lenta, causada pelo Mycobacterium leprae. A transmissão da doença ocorre pelas vias aéreas superiores (CDC, 2017). É determinante para a transmissão o contato próximo e prolongado com uma pessoa doente, com alta carga bacteriana e sem tratamento adequado, além da importância da imunocompetência de uma pessoa infectada para o desenvolvimento de uma infecção clínica após a transmissão (SANTOS KCB, et al., 2019; FISCHER M, 2017).

O diagnóstico é essencialmente epidemiológico e clínico, com base nas características dependendo do estágio clínico da doença, como na presença de máculas hipocrômicas hipoestésicas, placas infiltradas, infiltração, nódulos, tubérculos, alterações sensitivas e/ou motoras nos trajetos de troncos nervosos periféricos e/ou presença de bacilos de Hansen em amostras de esfregaço de pele (CHOPRA A, et al., 2019).

A doença possui caráter potencialmente incapacitante o qual pode levar a deformidades e a perda da função sensitiva e motora, sobretudo quando o diagnóstico é feito de forma tardia (SOUZA MF, et al., 2017). Tal fato se justifica devido ao patógeno ser um parasita intracitoplasmático de macrófagos, afetando o sistema tegumentar e os nervos periféricos, devido à predileção pelas células de Langerhans, células dendríticas encontradas na epiderme que possuem função de apresentadoras de antígenos aos linfócitos $T$, além de tropismo pelas células de Schwann, cuja função principal é permitir condução rápida dos impulsos nervosos além de isolar o axônio do compartimento extracelular (AZULAY RD, et al., 2013).

A infecção ativa por esse bacilo é caracterizada por grande diversidade de manifestações clínicas. Padronizou-se, a partir disso, classificações com intuito de agrupá-las, destacando duas: a Classificação Operacional e a Classificação de Madri. De acordo com a Classificação Operacional, os pacientes são divididos em paucibacilares (PB) pela presença de até cinco lesões e em multibacilares (MB) pela presença de seis ou mais lesões, porém o Ministério da Saúde (MS) também classifica como MB indivíduos que apresentam baciloscopia de pele positiva, sem considerar o número de lesões na pele (CAMPOS MRM, et al., 2018; BRASIL, 2017).

A Classificação de Madri, no entanto, utiliza características clínicas e baciloscópicas, dividindo em grupo instável, o qual inclui as formas Indeterminada e Dimorfa, e o grupo polar, composto pelas formas Tuberculoide e Virchowiana. O reconhecimento da classificação clínica é importante por auxiliar a escolha do tempo de tratamento (VELÔSO DS, et al., 2018).

O Brasil, nesse contexto, é classificado como um país de alta carga para a doença, ocupando o segundo lugar com relação a países com maior número de casos no mundo, mesmo após a introdução do esquema da poliquimioterapia (PQT), pela Organização Mundial da Saúde (OMS), constituído pela combinação de dapsona (DDS), clofazimina (CFZ) e rifampicina (RPM) para multibacilares, ter contribuído para a redução significativa na incidência mundial da doença, desde a década de 1980 (OMS, 2019; FRANCO MCA, et al., 2014). Destacam-se áreas hiperendêmicas no país, como o Estado do Pará, correspondente a uma taxa de detecção geral de casos novos de 30,44/100.000 habitantes, no ano de 2018 (BRASIL, 2020). 
O esquema de tratamento depende do perfil bacilífero e da faixa etária do paciente. Pacientes $\mathrm{PB}$, até o momento, ainda utilizam RMP associada à DDS durante 6 meses, caso a DSS precise ser suspensa, utilizase a CFZ em conjunto à RMP, pelo mesmo período. Os pacientes $M B$, no entanto, o tratamento de primeira linha é a associação de RMP, DDS e CFZ durante 12 meQses (PANTE CC, et al., 2018).

O tratamento, em crianças, baseia-se no peso corporal, porém seguindo a mesma sequência do esquema dos pacientes adultos. Os pacientes, durante o tratamento, devem tomar a dose diária proposta para cada medicamento, além de comparecer mensalmente para a administração da dose supervisionada (BRASIL, 2017). Em casos de intolerância ou contraindicação aos medicamentos do esquema padrão, o Ministério da saúde recomenda o tratamento alternativo nas unidades de referência, a depender do medicamento contraindicado e do perfil bacilífero, deve-se estabelecer a terapia substitutiva, introduzindo novos medicamentos, como Ofloxacino (OFX) e Minociclina (MNC) (BRASIL, 2019).

Os medicamentos que compõem o esquema terapêutico contra a hanseníase podem apresentar efeitos colaterais e como é um tratamento longo, os efeitos adversos podem ser o motivo do abandono do tratamento (FIGUEIREDO PV e HEINEN RC, 2017). A RPM, por exemplo, é um derivado da rifampicina $B$ e age por bloqueio da ácido ribonucleico (RNA) polimerase ácido desoxirribonucleico (DNA) dependente, impedindo a síntese de RNA mensageiro e de proteína pelo bacilo, apresentando efeitos adversos como hepatotoxicidade, trombocitopenia e anemia hemolítica, além da Síndrome Pseudogripal, caraterizada por febre, calafrios, astenia, cefaleia, mialgia, danos renais e, por fim, choque, ocorre devido à formação de anticorpos antirifampicina, decorrente do uso intermitente do medicamento (ARBEX MA, et al., 2010; BRASIL, 2019).

A DDS age inibindo a conversão de ácido para-aminobenzóico, consequentemente, o ácido fólico bacteriano não é formado. Distribuindo-se por todo o organismo, sendo mais notável na pele, no fígado e nos eritrócitos e, assim, inclui efeitos como metahemoglobinemia, fotodermatite e síndrome de Stevens-Johnson, ademais, pode ocorrer a Síndrome da Sulfona, complicação grave, definida por um quadro eritrodérmico descamativo súbito associado à sintomatologia sistêmica (LOPES MPQ, 2015; BRASIL, 2019). A CFZ, no entanto, liga-se ao DNA da micobactéria e ainda exerce uma atividade anti-inflamatória nas reações de eritema nodoso, é uma droga com caráter lipofílico, logo, tende-se acumular em tecidos adiposos e no sistema reticuloendotelial, sendo as reações mais importantes a hiperpigmentação cutânea, a ictiose e a síndrome do Intestino delgado (BRASIL, 2018).

Com base na relevância epidemiológica da região amazônica, o presente trabalho tem por objetivo avaliar a ocorrência de reações adversas à PTQ, para que se possa prevenir, informar ao paciente e diagnosticar precocemente a ocorrência de tais reações adversas, e assim o paciente tendo conhecimento das mesmas continue o tratamento de maneira adequada, reduzindo as chances de abandono.

\section{MÉTODOS}

Trata- se de um estudo observacional, descritivo e transversal. Foi utilizado um protocolo adaptado do estudo "Severe adverse reactions to multidrug therapy for leprosy, registered in tertiary services between 2012 and 2017 in Brazil", a fim de caracterizar os efeitos adversos relacionados ao tratamento da hanseníase. Além disso, foi implementado um protocolo de pesquisa próprio com as seguintes variáveis: a) identificação do paciente; b) classificação clínica e operacional da doença; c) baciloscopia; d) tratamento; e) testes laboratoriais antes e após o início do tratamento; f) história pessoal; g) dados relacionados a efeitos adversos à $P Q T$ e condutas.

O estudo foi realizado no ambulatório de dermatologia da Universidade do Estado do Pará, localizada em Belém, Pará, Brasil, centro de referência no tratamento de hanseníase no país. Este estudo foi aprovado pelo Comitê de Ética em Pesquisa (CEP) da Universidade do Estado do Pará, mediante parecer de número 3.853.243. Foi obtida uma amostra de 40 pacientes de ambos os sexos e com idade de 12 a 76 anos, no momento da consulta.

Os critérios de inclusão na pesquisa foram ter diagnóstico clínico e/ou diagnóstico complementar por biópsia e/ou por baciloscopia, encontrar-se em tratamento para hanseníase ou ter terminado a menos de 2 anos, consentir com a pesquisa e assinar o Termo de Consentimento Livre e Esclarecido (TCLE) para maiores 
de 18 anos e para as crianças e adolescentes, além do TCLE assinado pelos pais, obteve-se o Termo de Assentimento Livre e Esclarecido. Os critérios de exclusão foram os pacientes que não aceitaram participar da pesquisa, que abandonaram o tratamento para hanseníase, por motivos que não tenha sido reações adversas ao tratamento.

Os dados foram avaliados por estatística descritiva, utilizando medidas de tendência central, variância e frequências absoluta e relativa. O teste estatístico como Qui- quadrado para avaliação entre categorias e o Teste G para avaliação entre grupos. Toda inferência estatística foi realizada no software BioEstat 5.4, considerando $p$-valor significativo $\leq 0.05$.

\section{RESULTADOS}

Foram coletados dados de 40 pacientes onde destaca-se, na análise entre os dados sociodemográficos (Tabela 1), um predomínio de pacientes advindos da região metropolitana de Belém (55\%), com escolaridade de apenas ensino fundamental (70\%) e aposentados (35\%). Evidencia-se, quanto ao aspecto clínico da doença (Tabela 2), maior prevalência da forma Dimorfa (62,50\%), multibacilar (85\%) e com período de 12 meses de tratamento $(77,50 \%)$.

Tabela 1 - Características demográficas de pacientes com reações adversas à poliquimioterapia para hanseníase atendidos e serviço de referência, Belém, Pará, 2020.

\begin{tabular}{|c|c|c|c|}
\hline Características demográficas & $\mathbf{n}$ & $\%$ & p-valor \\
\hline \multicolumn{4}{|l|}{ Sexo } \\
\hline Feminino & 18 & 45,00 & \multirow{3}{*}{0.6353} \\
\hline Masculino & 22 & 55,00 & \\
\hline Total & 40 & 100,00 & \\
\hline \multicolumn{4}{|l|}{ Faixa etária } \\
\hline Menor de 15 anos & 2 & 5,00 & \multirow{8}{*}{0.1562} \\
\hline 15 a 30 anos & 4 & 10,00 & \\
\hline 31 a 40 anos & 7 & 17,50 & \\
\hline 41 a 50 anos & 9 & 22,50 & \\
\hline 51 a 60 anos & 7 & 17,50 & \\
\hline Maior de 60 anos & 11 & 27,50 & \\
\hline Média de idade \pm Desvio padrão & \multicolumn{2}{|c|}{$48,73 \pm 17.68$} & \\
\hline Total & 40 & 100,00 & \\
\hline \multicolumn{4}{|l|}{ Procedência } \\
\hline Região Metropolitana de Belém & 22 & 55,00 & \multirow{4}{*}{0.5218} \\
\hline Interior do Estado & 17 & 42,50 & \\
\hline Sem informação & 1 & 2,50 & \\
\hline Total & 40 & 100,00 & \\
\hline \multicolumn{4}{|l|}{ Escolaridade } \\
\hline Ensino Fundamental & 28 & 70,00 & \multirow{5}{*}{$<0.0001$} \\
\hline Ensino Médio & 8 & 20,00 & \\
\hline Ensino Superior & 1 & 2,50 & \\
\hline Sem informação & 3 & 7,50 & \\
\hline Total & 40 & 100,00 & \\
\hline \multicolumn{4}{|l|}{ Ocupação } \\
\hline Autônomo & 7 & 17,50 & \multirow{7}{*}{0.0269} \\
\hline Empregado & 9 & 22,50 & \\
\hline Desempregado & 5 & 12,50 & \\
\hline Aposentado & 14 & 35,00 & \\
\hline Estudante & 2 & 5,00 & \\
\hline Sem informação & 3 & 7,50 & \\
\hline Total & 40 & 100,00 & \\
\hline
\end{tabular}

Fonte: Pires CAA, et al., 2020. 
Tabela 2 - Características clínicas de pacientes com reações adversas à poliquimioterapia para hanseníase atendidos em serviço de referência, Belém, Pará, 2020.

\begin{tabular}{lccc}
\hline & $\mathbf{n}$ & $\%$ & p-valor \\
\hline Classificação Clínica & & & \\
\hline Dimorfa & 25 & 62,50 & 0.0004 \\
Tuberculóide & 6 & 15,00 & 22,50 \\
Virchowiana & 9 & 100,00 & $<0.0001$ \\
Total & 40 & & \\
\hline Classificação operacional & & 15,00 & \\
\hline Paucibacilar & 6 & 85,00 & $<0.0001$ \\
Multibacilar & 34 & 100,00 & \\
Total & 40 & & \\
\hline Tempo de tratamento & & 15,00 & \\
\hline 6 meses & 6 & 77,50 & \\
12 meses & 31 & 7,50 & \\
24 meses & 3 & 100,00 & \\
Total & 40 & & \\
\hline
\end{tabular}

Fonte: Pires CAA, et al., 2020.

Dentre as reações adversas associadas aos medicamentos (Tabela 3) e aos sistemas acometidos pela medicação (Tabela 4), ressalta-se a mialgia $(65 \%)$ e cefaleia $(47,50 \%)$ com relação à Dapsona. Quanto à Rifampicina, predominantemente, queixas gastrointestinais, em primeiro lugar enjoo (32,50\%), seguido de vômito (15\%), $5 \%$ dos pacientes que utilizaram essa medicação alegaram alteração no sistema excretor. Os efeitos colaterais mais prevalentes da Clofazimina, entretanto, foram hiperpigmentação cutânea $(77,50 \%)$ e ictiose $(62,5 \%)$, com a ressalva de que todos os pacientes em uso da medicação (100\%) apresentaram algum tipo de alteração do sistema tegumentar.

Tabela 3 - Reações adversas para dapsona, rifampicina e clofazimina durante poliquimioterapia para hanseníase $(n=40)$ atendidos e serviço de referência, Belém, Pará, 2020.

\begin{tabular}{|c|c|c|c|c|c|c|c|c|c|}
\hline \multirow{3}{*}{ Reações } & \multicolumn{4}{|c|}{ Sexo } & \multicolumn{4}{|c|}{ Faixa etária } & \multirow{3}{*}{ p-valor } \\
\hline & \multicolumn{2}{|c|}{ Masculino } & \multicolumn{2}{|c|}{ Feminino } & \multicolumn{2}{|c|}{ Até 59 anos } & \multicolumn{2}{|c|}{60 anos ou mais } & \\
\hline & $\mathbf{N}$ & $\%$ & $\mathrm{n}$ & $\%$ & $\mathbf{n}$ & $\%$ & $\mathrm{n}$ & $\%$ & \\
\hline \multicolumn{10}{|l|}{ Dapsona } \\
\hline Episgastralgia & 2 & 5,00 & 8 & 20,00 & 7 & 17,50 & 3 & 7,50 & \\
\hline Cefaléia & 10 & 25,00 & 9 & 22,50 & 16 & 40,00 & 3 & 7,50 & \\
\hline Fotodermatite & 2 & 5,00 & - & - & 2 & 5,00 & - & - & \\
\hline Eritema/edema & 8 & & 7 & 17,50 & 14 & 35,00 & 1 & 2,50 & \\
\hline Mialgia & 13 & 32,50 & 13 & 32,50 & 21 & 52,50 & 5 & 12,50 & ${ }^{*} 0.2976$ \\
\hline Neurite & 2 & 5,00 & 1 & 2,50 & 3 & 7,50 & - & - & ${ }^{* *} 0.7138$ \\
\hline Oligúria & 5 & 12,50 & 1 & 2,50 & 5 & 12,50 & 1 & 2,50 & \\
\hline Hiporexia & 5 & 12,50 & 7 & 17,50 & 8 & 20,00 & 4 & 10,00 & \\
\hline Insônia & 5 & 12,50 & 5 & 12,50 & 7 & 17,50 & 3 & 7,50 & \\
\hline Dispnéia & 2 & 5,00 & 5 & 12,50 & 5 & 12,50 & 2 & 5,00 & \\
\hline \multicolumn{10}{|l|}{ Rifampicina } \\
\hline Enjoo & 6 & 15,00 & 7 & 17,50 & 10 & 25,00 & 3 & 7,50 & \\
\hline Diarreia & 1 & 2,50 & 1 & 2,50 & 1 & 2,50 & 1 & 2,50 & \\
\hline Vômito & 3 & 7,50 & 3 & 7,50 & 6 & 15,00 & - & - & ${ }^{*} 0.3072$ \\
\hline Febre & 1 & 2,50 & 1 & 2,50 & 1 & 2,50 & 1 & 2,50 & ${ }^{* \star} 0.3897$ \\
\hline Cólica renal & - & 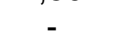 & 5 & 12,50 & 3 & 7,50 & 2 & 5,00 & \\
\hline \multicolumn{10}{|l|}{ Clofazimina } \\
\hline Ardência & 4 & 10,00 & 6 & 15,00 & 6 & 15,00 & 4 & 10,00 & \\
\hline Prurido & 7 & 17,50 & 5 & 2,50 & 8 & 20,00 & 4 & 10,00 & \\
\hline Ictiose & 14 & 35,00 & 11 & 27,50 & 17 & 42,50 & 8 & 20,00 & ${ }^{*} 0.6640$ \\
\hline Hiperpigmentação cutânea & 17 & 42,50 & 14 & 35,00 & 21 & 52,50 & 10 & 25,00 & ${ }^{* *} 0.9195$ \\
\hline Xerodermia & - & - & 1 & 2,50 & 1 & 2,50 & - & - & \\
\hline Xeroftalmia & 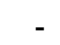 & 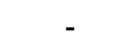 & 1 & 2,50 & 1 & 2,50 & - & & \\
\hline
\end{tabular}

Legenda: *Teste $\mathrm{G}$ aplicado entre os grupos Masculino e Feminino. **Teste $\mathrm{G}$ aplicado entre os grupos até 59 anos e 60 anos ou mais.

Fonte: Pires CAA, et al., 2020. 
Tabela 4 - Sistemas afetados por reações adversas para poliquimioterapia em pacientes de hanseníase $(\mathrm{n}=40)$ atendidos em serviço de referência, Belém, Pará, 2020.

\begin{tabular}{lccccccc}
\multicolumn{1}{c}{ Sistema } & \multicolumn{2}{c}{ Dapsona } & \multicolumn{2}{c}{ Rifampicina } & \multicolumn{2}{c}{ Clofazimina } & \multirow{2}{*}{ p-valor } \\
\cline { 2 - 7 } & $\mathbf{n}$ & $\%$ & $\mathbf{n}$ & $\%$ & $\mathbf{n}$ & $\%$ & \\
\hline Excretor & 6 & 15,00 & 2 & 5,00 & - & - & \multirow{2}{*}{$<0.0001$} \\
Nervoso periférico & 2 & 5,00 & - & - & - & - & \\
Tegumentar & 2 & 5,00 & - & - & 40 & 100,00 & \\
Respiratório & 7 & 17,50 & - & - & - & - & \\
\hline
\end{tabular}

Fonte: Pires CAA, et al., 2020.

A respeito à ocorrência de reações adversas segundo o uso de outras medicações, devido às comorbidades prévias do paciente, tais como hipertensão arterial sistêmica ou reações hansênicas, e seu tempo de uso (Figura 1), nota-se uma frequência semelhante entre as três medicações da Poliquimioterapia. Não foi encontrada nenhuma variável estatisticamente significativa entre as correlações analisadas.

Figura 1 - Ocorrência de reações adversas durante o uso de poliquimioterapia em pacientes de hanseníase $(n=40)$ segundo o uso de outras medicações e tempo de uso, atendidos em serviço de referência, Belém, Pará, 2020.

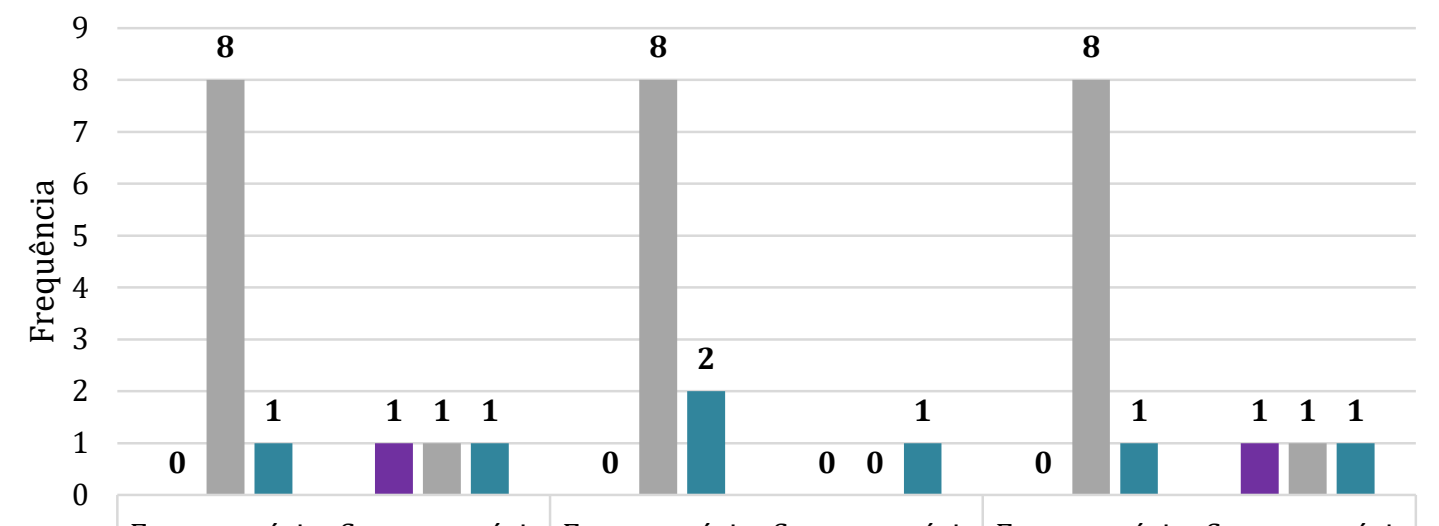

Em uso prévio Sem uso prévio Em uso prévio Sem uso prévio Em uso prévio Sem uso prévio

Dapsona

Rifampicina

Clofazimina

- Menos de 1 ano $\quad 1$ a 5 anos $\square$ Mais de 5 anos

Fonte: Pires CAA, et al., 2020.

\section{DISCUSSÃO}

A poliquimioterapia é extremamente vantajosa no controle da hanseníase, dessa maneira, a adesão e completa realização do tratamento são fundamentais para a quebra de transmissão e o combate à doença. O tratamento da hanseníase, todavia, é considerado longo e as drogas utilizadas apresentam efeitos colaterais proeminentes, a partir disso, pressupõe-se que isso seja um dos motivos para o abandono do tratamento, evidenciado pela ausência da tomada de dose supervisionada de PQT (FIGUEIREDO PV e HEINEN RC, 2017).

Observa-se, com relação ao perfil epidemiológico, este segue o padrão nacional em que há maior prevalência no sexo masculino $(55,2 \%)$, o que pode estar associado à maior movimentação e oportunidade de contato social entre homens devido às atividades laborais fora do domicílio, ou pode-se pensar na teoria que refere que a maior propensão dos indivíduos do sexo masculino desenvolverem hanseníase, uma vez que a testosterona pode facilitar a infecção induzindo resposta do tipo TH2, necessitando, por conseguinte, de tratamento (BRASIL, 2020; NOBRE ML, et al., 2017).

Quanto a classificação operacional, evidenciou-se maior número de indivíduos com as formas multibacilares e de pacientes com média de idade de 48,73 anos, demonstrando o predomínio de pacientes em fases economicamente ativas, haja vista que esse grupo possui maior convivência social e, 
consequentemente, são mais suscetíveis a contrair a hanseníase, tal fato pode repercutir, a longo prazo, na economia de um país de uma maneira geral (OLIVEIRA JSS, et al., 2019; GNIMAVO RS, et al., 2020). Ademais a média de idade elevada, pode estar relacionada a um período de incubação mais prolongado ou, ainda, a diagnósticos mais demorados os quais postergariam a instituição correta do tratamento, contribuindo para a manutenção de casos não diagnosticados, que poderão progredir para deformidades e incapacidades, além da continuidade da cadeia de transmissão (MARTINS RJ, et al., 2016).

A ausência desses pacientes em suas atividades profissionais, por motivos de efeitos adversos à PTQ, associados ao tempo destinado ao acompanhamento mensal, acarreta em possíveis demissões, que podem desencadear problemas econômicos, com um imenso custo social (FIGUEIREDO PV e HEINEN RC, 2017). A PQT, outrossim, como outros tratamentos quimioterápicos, pode aumentar as chances de morbidade e até a mortalidade se o diagnóstico de um efeito adverso for feito de forma tardia (ROCHA MCN, et al., 2015). É de suma importância, a partir disso, treinar a equipe de saúde envolvida no cuidado integral do paciente quanto às principais reações adversas durante o tratamento e como manejar tais situações, além de esclarecer o paciente e os familiares sobre o assunto, conscientizando sobre o não abandono, ajudando, em consequência, na adesão ao tratamento, medida eficaz para diminuir a cadeia de transmissão da hanseníase (ROLIM MFN, et al., 2016).

A dapsona, um dos fármacos que compõem a PQT no tratamento para a hanseníase, é metabolizada no fígado, sendo responsável pela produção de hidroxilamina, e pelas alterações hematológicas do medicamento, como a metahemoglobina e anemia hemolítica (CARVALHO C, et al., 2011). O presente estudo não observou pacientes com estes quadros, os quadros de anemia identificados nos pacientes não foram caracterizados como hemolítica. Ressalta-se, a importância do acompanhamento laboratorial do paciente e o registro obrigatório nos prontuários para possível detecção precoce de algumas reações indesejáveis (FONSECA HG, et al., 2020).

Quando considerada as reações adversas provenientes do uso de dapsona, observou-se predomínio de sintomas relacionados ao mal-estar geral, como mialgia, cefaleia e hiporexia, semelhante aos dados descritos na literatura (KARJIGI S, et al., 2016). A "Síndrome da Sulfona", também denominada de Síndrome de Hipersensibilidade à Dapsona, é um dos efeitos adversos mais graves da medicação, caracterizada por eosinofilia e sintomas sistêmicos, como: febre, mal-estar, mialgias, linfoadenomegalia generalizada, hepatomegalia e "rash" cutâneo, que se manifestam entre 2 a 5 semanas após o início do tratamento, sendo potencialmente fatal, caso não tratada adequadamente (LIU H, et al., 2019). No presente estudo, ocorreu um caso desta síndrome, em um paciente portador do Vírus da Imunodeficiência Humana (HIV) em uso de terapia antirretroviral, o que motivou a mudança para o esquema alternativo, tendo evoluído satisfatoriamente para a cura bacteriológica completando o esquema alternativo com a substituição da dapsona pela ofloxacina sem novas intercorrências.

Nesses casos de intolerância à DDS, o Ministério da Saúde recomenda aos indivíduos PB fazerem o uso de RMP 600mg, CFZ 300mg doses mensais e supervisionadas e CFZ 50mg dose diária autoadministrada com 6 doses em até 9 meses. É indicado, aos pacientes MB nessa situação, o uso de RMP 600mg, CFZ 300mg e Ofloxacino (OFX) 400mg dose mensal supervisionada com doses diárias autoadministradas de OFX 400mg, CFZ 50mg ou Minociclina (MNC) 100mg dose mensal supervisionada e doses diárias autoadministradas também de $100 \mathrm{mg}$, com tratamento de 12 doses e duração de até 18 meses (BRASIL, 2019).

O uso da clofazimina possui boa tolerabilidade e suas reações adversas estão ligadas à dose, sendo frequentemente reversíveis com a descontinuação da medicação (MAIA MV, et al., 2013). As reações adversas observadas, provenientes da aplicação da mesma, foram principalmente pigmentação cutânea alterada (marrom-avermelhada) e ictiose, que se acentuam com o sol, deixando-a mais ressecada e sujeita a eczematizações, ocorre devido ao acúmulo de pigmento nos macrófagos. A pele ictiósica ocorre em quase todos os pacientes sob tratamento com esta medicação, a depender da dose e do tipo de pele (KABUTA RMM, et al., 2014). É necessário, à vista disso, alertar os pacientes em uso desse medicamento, para evitarem a exposição à luz solar e realizar uma hidratação intensiva da pele. 
O efeito adverso mais grave da clofazimina decorre do depósito de cristais que podem se acumular em diversos órgãos, tais quais histiócitos da mucosa intestinal e linfonodos mesentéricos. Essa deposição pode levar à infecção intestinal e obstrução intestinal, sendo necessário, em certos casos, até mesmo indicar uma laparotomia (BRASIL, 2018). Logo, pacientes com queixas abdominais, como náuseas e diarreias, devem ser acompanhados clinicamente e com possível redução ou suspensão da medicação (NOVARTIS, 2016). No estudo, as queixas abdominais foram relatadas devido ao uso das demais medicações, rifampicina e dapsona, e não à clofazimina em si, não possuindo casos severos como o descrito.

A maioria dos efeitos colaterais no tratamento poliquimioterápico ocorre devido ao uso da rifampicina, no entanto, o presente estudo diverge da literatura, uma vez que não foram registrados muitos efeitos adversos com relação ao uso dessa droga e o mais frequentemente descrito foi o enjoo, seguido de vômitos e cólica renal (BRASIL, 2018). Outras manifestações podem vir a ocorrer, como as máculas eritematosas na face e couro cabeludo após 2 a 3 horas após a ingestão (FISCHER M, 2017).

Os efeitos colaterais graves são observados, em especial, em caso de uso abusivo de álcool ou em casos que a droga é administrada intermitente, estes incluem hepatotoxicidade com testes de função hepática elevados e colestase intra-hepática (FISCHER M, 2017). Essas reações imunoalérgicas são mediadas por anticorpos IgG e IgM, estes, por sua vez, acometem plaquetas, eritrócitos e outras células, tais quais as células epiteliais tubulares renais, podendo desenvolver, respectivamente, trombocitopemia imune-mediada, anemia hemolítica e insuficiência renal aguda (FRANCO LA, 2014).

Outras reações adversas podem ocorrer, devido ao uso de outras medicações utilizadas pelos pacientes, levando a uma interação medicamentosa com as medicações da PQT, ainda pouco descrita na literatura (SILVA DLG, et al., 2019). A presente pesquisa analisou, de forma geral, pacientes que faziam uso de outras medicações para comorbidades prévias, evidenciando o aumento da propensão à ocorrência de reações adversas, porém sem relação estatisticamente significativa. Nesse sentido, alguns pacientes podem ter apresentado sobreposição de sintomas adversos pelo uso concomitante, por exemplo, de medicamentos comumente utilizados pela população como metformina, hidroclorotiazida, anti-inflamatórios não esteroides e omeprazol podem causar hemólise, efeito adverso no tratamento da hanseníase devido à dapsona (DIPIRO JT, et al., 2014). Além disso, a maioria das drogas supracitadas, apesar de diferentes mecanismos de ação, produzem efeitos colaterais gastrointestinais, tais como hepatotoxicidade e náuseas, semelhantes aos efeitos da rifampicina (CERQUEIRA SRPS, et al., 2020).

Ressalta-se, ainda que na hanseníase podem ocorrer os estados reacionais, onde mais medicações são acrescentadas, como no caso do uso da talidomida, medicação que é comumente prescrita para reação hansênica tipo II, complicação não rara da hanseníase. Esta pode ocasionar edema de extremidades, anormalidades oculares e auriculares, neuropatia periférica, prurido, ressecamento de pele e de mucosa (DRUMMOND PLM, et al., 2019). Ademais, corticosteroides orais também podem ser usados, para neurites, reações tipo I e ainda em conjunto à talidomida, para o tratamento de eritema nodoso hansênico (ENL), por gerar alterações na função endotelial, aumentando a secreção de interferon-gama e interleucina-2 (SHARMA NL, et al., 2004). Diante disso, eventos tromboembólicos foram descritos no tratamento de ENL com talidomida e corticosteroide, não estando relacionado a eventos genéticos de trombofilia, mas sim a associação dos dois medicamentos (PORTO LAB, et al., 2019).

\section{CONCLUSÃO}

As reações adversas mais frequentes, no estudo, estão relacionadas à Dapsona, através de manifestações gastrointestinais e sistêmicas inespecíficas, e à Clofazimina, por meio da hiperpigmentação cutânea e da ictiose, sendo que a maioria dos pacientes apresentaram efeitos colaterais adversos. Esses efeitos têm importância com relação ao abandono do tratamento, é fundamental, logo, a realização de exames laboratoriais periódicos junto às consultas, para a detecção precoce dos efeitos adversos à PQT, a fim de antecipar a intervenção em eventos potencialmente graves. Tal fato proporciona melhorias na qualidade de vida do paciente e mais segurança à equipe de saúde, resultando em um controle da hanseníase, colaborando para o alcance da meta de eliminar essa doença como um problema de saúde pública a nível nacional. 


\section{REFERÊNCIAS}

1. ARBEX MA, et al. Antituberculosis drugs: Drug interactions, adverse effects, and use in special situations. Part 1: Firstline drugs. Jornal Brasileiro de Pneumologia, 2010; 36 (5):626-640.

2. AZULAY RD, et al. Dermatologia. 6 ed. Rio de Janeiro: Guanabara Koogan, 2013; 949-1018p.

3. BRASIL, Ministério da Saúde. Secretaria de Ciência, Tecnologia e Insumos Estratégicos. Ampliação do uso da clofazimina para hanseníase paucibacilar. Brasília, 2018.

4. BRASIL, Ministério da Saúde. Secretaria de Vigilância em Saúde, Coordenação-Geral de Desenvolvimento da Epidemiologia em Serviços. Guia de Vigilância em Saúde. 4 ed. Brasília, 2019.

5. BRASIL, Ministério da Saúde. Secretaria de Vigilância em Saúde, Departamento de Vigilância das Doenças Transmissíveis. Guia Prático Sobre a Hanseníase. 1 ed. Brasília, 2017.

6. BRASIL, Ministério da Saúde. Secretaria de Vigilância em Saúde. Departamento de Doenças de Condições Crônicas e Infecções Sexualmente. Boletim Epidemiológico de Hanseníase. 1 ed. Brasília, 2020.

7. BRASIL, Ministério da Saúde. Taxa de detecção de novos casos de hanseníase em menores de 15 anos: Taxa de Detecção de hanseníase em menores de 15 anos por 100.000 habitantes Estados e regiões, Brasil, 1994 a 2017. SINAN. 2018.

8. CAMPOS MRM, et al. Perfil Clínico-Epidemiológico dos Pacientes Diagnosticados com Hanseníase na Paraíba e no Brasil, 2008-2012. Revista Brasileira de Ciências da Saúde, 2018; 22(1): 83-90.

9. CARVALHO C, et al. Methemoglobinemia: case report and review. Arquivos de Medicina, 2011; 25 (3).

10. CDC (CENTERS FOR DISEASE CONTROL AND PREVENTION). Hansen's disease (leprosy), 2017.

11. CERQUEIRA SRPS, et al. The interference of polypharmacy and the importance of clinical pharmacy advice in the treatment of leprosy: a case-control study. Revista da Sociedade Brasileira de Medicina Tropical, 2020; 53.

12. CHOPRA A, et al. Correlation of Dermoscopic and Histopathologic Patterns in Leprosy - A Pilot Study. Indian Dermatology Online Journal, 2019; 10(6): 663-668.

13. DIPIRO JT, et al. Pharmacotherapy: A Pathophysiologic Approach. 9e. New York, NY: McGraw-Hill; 2014.

14. DRUMMOND PLM, et al. Adverse events in patients with leprosy on treatment with thalidomide. Revista da Sociedade Brasileira de Medicina Tropical; 2019; 52.

15. FONSECA HG, et al. Systematization of nursing care to an elderly with leprosy. Brazilian Journal of Development, 2020; 6 (6): 40843-40853.

16. FISCHER M. Leprosy - an overview of clinical features, diagnosis, and treatment. Journal of the German Society of Dermatology, 2017; 5 (8): 801-827.

17. FRANCO LA. Reações adversas à poliquimiterapia em hanseníase. Universidade Federal de Sergipe, Aracajú, SE, 2014.

18. FRANCO MCA, et al. Perfil de casos e fatores de risco para hanseníase, em menores de quinze anos, em um município hiperendêmico da Região Norte do Brasil. Revista Paraense de Medicina, 2014; 28 (4).

19. GNIMAVO RS, et al. Trends of the leprosy control indicators in Benin from 2006 to 2018. BMC Public Health, 2020; 20(1):1254.

20. FIGUEIREDO PV e HEINEN RC. Poliquimioterapia no tratamento da hanseníase. Revista Saúde Física \& Mental, 2017; 5 (2).

21. KABUTA RMM, et al. Efeitos adversos da poliquimioterapia para hanseníase: utilização de doses alternativas e avaliação pós alta. Hansenologia Internationalis, 2014; 39 (1): 8-21.

22. KARJIGI S, et al. Dapsone: An Update. Indian Journal of Leprosy, 2016; 87(4): 233-239.

23. LOPES MQP. Avaliação dos fenótipos de aceitação e hidroxilação predominantes nas populações de 5 macrorregiões do Brasil baseada no genomapossível influência da farmacogenética na conduta terapêutica da hanseníase. Instituto Oswaldo Cruz, Rio de Janeiro, RJ, 2015.

24. LIU H, et al. Evaluation of Prospective HLA-B*13:01 Screening to Prevent Dapsone Hypersensitivity Syndrome in Patients With Leprosy. JAMA Dermatology, 2019; 155(6): 666-672.

25. MAIA MV, et al. Efeitos adversos das drogas (minociclina, ofloxacina e clofazimina) utilizadas no esquema alternativo para pacientes com hanseníase multibacilar, em unidade de referência, Manaus, Amazonas, Brasil. Anais Brasileiros de Dermatologia, 2013; 88 (2):205-210.

26. MARTINS RJ, et al. Sociodemographic and epidemiological profile of leprosy patients in an endemic region in Brazil. Revista da Sociedade Brasileira de Medicina Tropical, 2016; 49: 6.

27. NOBRE ML, et al. Multibacillary leprosy by population groups in Brazil: Lessons from an observational study. Plos Neglected Tropical Diseases, 2017; 11(2).

28. NOVARTIS PHARMACEUTICALS CORPORATION: Lamprene. Label, 2016.

29. OLIVEIRA JSS, et al. Leprosy in elderly people and the profile of a retrospective cohort in an endemic region of the Brazilian Amazon. Plos Neglected Tropical Diseases, 2019; 13 (9).

30. OMS (ORGANIZAÇÃO MUNDIAL DA SAÚDE). Global leprosy update, 2018: moving towards a leprosy free world. Weekly Epidemiological Record, Genebra, n. 94, p. 389-412, 30 ago. 2019.

31. PANTE CC, et al. Severe adverse reactions to multidrug therapy for leprosy, registered in tertiary services between 2012 and 2017 in Brazil. Lepr Rev, 2018; 80 (4): 328-334.

32. PODDER I, et al. Clinical and Histopathological Response to Multidrug Therapy in Paucibacillary Leprosy at the end of 6 Months: A Prospective Observational Study from Eastern India. Indian Journal of Dermatology, 2018; 63(1): 47-52.

33. PORTO LAB, et al. Deep Venous Thrombosis in Patients with Erythema Nodosum Leprosum in the Use of Thalidomide and Systemic Corticosteroid in Reference Service in Belo Horizonte, Minas Gerais. Case Reports in Dermatological Medicine, 2019. 
34. ROCHA MCN, et al. Óbitos registrados com causa básica hanseníase no Brasil: uso do relacionamento de bases de dados para melhoria da informação. Ciência \& Saúde Coletiva, 2015; 20 (4).

35. ROLIM MFN, et al. Fatores relacionados ao abandono ou interrupção do tratamento da Hanseníase. Journal of medicine and Health Promotion, 2016; 1 (3).

36. SANTOS KCB, et al. Strategies for control and surveillance of leprosy contacts: integrative review. Saúde em Debate, 2019; 43: 576-591.

37. SHARMA NL, et al. Deep vein thrombosis: a rare complication of thalidomide therapy in recurrent erythema nodosum leprosum. Internacional Journal of Leprosy, ,2004; 72 (4): 483-5.

38. SILVA DLG, et al. Novas perspectivas do diagnóstico e tratamento da hanseníase. Revista Referências em Saúde da Faculdade Estácio de Sá de Goiás- RRS-FESGO, 2019; 2 (3): 75-81.

39. SOUZA MF, et al. Avaliação da implantação do Programa de Controle da Hanseníase em Camaragibe, Pernambuco. Epidemiologia e Serviços de Saúde, 2017; 26: 817-834.

40. VELÔSO DS, et al. Perfil Clínico Epidemiológico da Hanseníase: Uma Revisão Integrativa. REAS: Revista Eletrônica Acervo Saúde, 2018; 10: 1-9. 